\title{
DYNAMICS OF A GENERALIZED COSMOLOGICAL SCALAR-TENSOR THEORY
}

\author{
TAKAO FUKUI JAMES M. OVERDUIN \\ Department of Physics, University of Waterloo \\ Waterloo, Ontario, Canada N2L $3 G 1$
}

\begin{abstract}
A generalized scalar-tensor (GST) theory is investigated whose cosmological (or quintessence) term depends on both a scalar field and its time derivative. A correspondence with solutions of five-dimensional Space-Time-Matter (STM) theory is noted. Analytic solutions are found for the scale factor, scalar field and cosmological term. Models with free parameters of order unity are consistent with recent observational data and could be relevant to both the dark matter and cosmological "constant" problems.
\end{abstract}

\section{Introduction}

Generalized scalar-tensor (GST) theoriest have enjoyed new attention in recent years, partly because the potential energy of the scalar field and/or the presence of a variable cosmological term could drive inflation, resolving puzzles such as the monopole, horizon and flatness problems 3 The variable cosmological term has also been mentioned as a possible solution to the cosmological "constant" problem, 3 and - most recently - as a candidate for the dark matter (or quintessence) making up most of the Universe. 1

In a previous paper ${ }^{3}$ we have introduced a modified GST theory in which the cosmological term $\Lambda$ depends not only on the scalar field $\phi$ but its gradient $\phi_{, i} \phi^{, i}$ as well. A correspondence with solutions of Wesson's five-dimensional Space-TimeMatter (STM) theory 6 has been observed, with the scalar field arising in fourdimensional spacetime as a manifestation of higher-dimensional geometry. Here we obtain cosmological solutions of the theory and explore their properties in detail, with special regard to constraints from recent observational data.

\footnotetext{
*E-mail: fukui@sciborg.uwaterloo.ca

${ }^{\dagger}$ Permanent address: Department of Language and Culture, Dokkyo University, Soka, Saitama 340-0042, Japan

${ }^{\ddagger}$ Current address: Astrophysics and Cosmology Group, Department of Physics, Waseda University, Okubo 3-4-1, Shinjuku-ku, Tokyo 169-8555, Japan
} 
The theory is summarized and applied to cosmology (the Robertson-Walker metric) in Section 2. The connection to STM theory is drawn in Section 3, where solutions for the scale factor $a$, Hubble parameter $H$ and deceleration parameter $q$ are discussed. Analytic expressions for the scalar field and cosmological term are derived in Sections 4 and 5, and related to observation via the energy density parameters $\Omega_{m, 0}$ and $\Omega_{\Lambda, 0}$. Section 6 is a discussion.

\section{Modified GST Cosmology}

We consider here a modified GST theory, first introduced by the authors in Ref. 5 , in which $\Lambda$ depends on both the scalar field $\phi$ and its gradient $\phi_{, i} \phi^{i}$ (the Brans-Dicke coupling parameter $\omega$ is however taken as a function of $\phi$ alone). This dependence leads to significant departures from earlier GST theories in which $\Lambda=\Lambda(\phi)$ only Possible implications for the early universe have recently been investigated in Ref. 8 . In this paper we focus primarily on the later stages of cosmic evolution (i.e., the radiation and matter-dominated eras).

The action principle of the modified GST theory is

$$
0=\delta \int\left\{\phi\left[R+2 \Lambda\left(\phi, \phi_{, i} \phi^{, i}\right)\right]+\frac{16 \pi}{c^{4}} L_{m}-\omega(\phi) \frac{\phi_{, i} \phi^{, i}}{\phi}\right\} \sqrt{-g} d \Omega
$$

where the Latin index $i$ runs over $0,1,2$ and 3 . Variation leads to the following field equation for the metric field $g_{i j}$

$$
R_{i j}-\frac{1}{2} g_{i j} R=\frac{8 \pi}{\phi c^{4}}\left(T_{i j}+T_{i j}^{\phi}\right)
$$

where $b \equiv \phi_{, l} \phi^{l}, T_{i j}$ is the energy-momentum tensor of matter, and the energymomentum tensor of the scalar field is defined as follows

$$
\frac{8 \pi}{\phi c^{4}} T_{i j}^{\phi} \equiv \frac{\omega}{\phi^{2}}\left(\phi_{, i} \phi_{, j}-\frac{1}{2} g_{i j} b\right)+\frac{1}{\phi}\left(\phi_{, i ; j}-g_{i j} \square \phi\right)+g_{i j} \Lambda-2 \frac{\partial \Lambda}{\partial b} \phi_{, i} \phi_{, j} .
$$

When $\phi=$ const., $T_{i j}^{\phi}$ reduces to the vacuum energy-momentum tensor $T_{i j}^{\mathrm{vac}}=\Lambda g_{i j}$, as expected. The field equation for $\phi$ is

$$
R+2 \Lambda+2 \phi \frac{\partial \Lambda}{\partial \phi}-4 \frac{\partial}{\partial x^{l}}\left(\phi \frac{\partial \Lambda}{\partial b}\right) \phi^{, l}-4 \phi \frac{\partial \Lambda}{\partial b} \square \phi=\frac{\omega}{\phi^{2}} b-\frac{2 \omega}{\phi} \square \phi-\frac{b}{\phi} \frac{d \omega}{d \phi} .
$$

Eq. (4) ensures that the conservation law $T_{i ; k}^{k}=0$ holds, as required.

When the scalar field $\phi$ depends only on $x^{0}=c t$, then the field equations for homogeneous and isotropic perfect-fluid cosmology may be obtained from Eq. (2) as follows 3 The time-time component gives

$$
\left(\frac{\dot{a}}{a}\right)^{2}+\frac{k c^{2}}{a^{2}}-\frac{\Lambda c^{2}}{3}+\frac{2}{3} \frac{\partial \Lambda c^{2}}{\partial \dot{\phi}^{2}} \dot{\phi}^{2}=\frac{8 \pi}{3 \phi c^{2}} \epsilon+\frac{\omega}{6}\left(\frac{\dot{\phi}}{\phi}\right)^{2}-\frac{\dot{a}}{a} \frac{\dot{\phi}}{\phi},
$$


while the space-space components lead to

$$
2 \frac{\ddot{a}}{a}+\left(\frac{\dot{a}}{a}\right)^{2}+\frac{k c^{2}}{a^{2}}-\Lambda c^{2}=-\frac{8 \pi}{\phi c^{2}} p-\frac{\omega}{2}\left(\frac{\dot{\phi}}{\phi}\right)^{2}-\frac{\ddot{\phi}}{\phi}-2 \frac{\dot{a}}{a} \frac{\dot{\phi}}{\phi} .
$$

From Eq. (雨), we havea

$$
\begin{aligned}
\frac{\ddot{a}}{a}+\left(\frac{\dot{a}}{a}\right)^{2} & +\frac{k c^{2}}{a^{2}}-\frac{\Lambda c^{2}}{3}-\frac{\phi}{3} \frac{\partial \Lambda c^{2}}{\partial \phi}+\frac{2}{3} \frac{\partial}{\partial t}\left(\phi \frac{\partial \Lambda c^{2}}{\partial \dot{\phi}^{2}}\right) \dot{\phi}+\frac{2}{3} \phi \frac{\partial \Lambda c^{2}}{\partial \dot{\phi}^{2}}\left(\ddot{\phi}+3 \frac{\dot{a}}{a} \dot{\phi}\right) \\
& =\omega \frac{\dot{a}}{a} \frac{\dot{\phi}}{\phi}+\frac{\omega}{3} \frac{\ddot{\phi}}{\phi}+\frac{1}{6} \frac{d \omega}{d \phi} \frac{\dot{\phi}^{2}}{\phi}-\frac{\omega}{6}\left(\frac{\dot{\phi}}{\phi}\right)^{2} .
\end{aligned}
$$

This equation can be greatly simplified if the cross-terms in $a$ and $\phi$ on either side are equated. In particular the ansatz

$$
\frac{\partial \Lambda c^{2}}{\partial \dot{\phi}^{2}}=\frac{\omega}{2 \phi^{2}},
$$

reduces Eq. (7) to the separable form

$$
\frac{\ddot{a}}{a}+\left(\frac{\dot{a}}{a}\right)^{2}+\frac{k c^{2}}{a^{2}}=\frac{1}{3}\left[\Lambda c^{2}+\phi \frac{\partial \Lambda c^{2}}{\partial \phi}-\frac{1}{2} \frac{d \omega}{d \phi} \frac{\dot{\phi}^{2}}{\phi}+\frac{\omega}{2}\left(\frac{\dot{\phi}}{\phi}\right)^{2}\right] .
$$

This is natural because one expects that $a$ (a metric quantity) and $\phi$ (part of the source term $T_{i j}^{\phi}$ ) should be independent. In what follows, we use Eqs. (5), (6) and (9) with the ansatz (8) to solve for $a, \phi$ and $\Lambda c^{2}$.

\section{Connection with STM Theory and Evolution of the Scale Factor}

Both sides of Eq. (9) may be equated to a parameter $K$ which is taken to be constant according to the arguments of Ref. 8. We focus in this paper on the case $K=0$; the complementary situation in which $K \neq 0$ has been examined in Ref. 8. The choice $K=0$ is motivated by STM theory, a generalization of Kaluza-Klein theory in which Kaluza's "cylinder condition" is relaxed to allow dependence on the extra coordinate(s) in principle. A large literature 6 has now built up around this theory, in which 4D field equations of the form (2), together with a scalar field $\phi$ and a wide class of $4 \mathrm{D}$ matter fields, can be "induced" from pure geometry in $5 \mathrm{D}$; that is, from the action principle $0=\delta \int R^{(5)} \sqrt{-g^{(5)}} d^{5} x$ (or, equivalently, the vacuum field equations $R_{A B}^{(5)}=0$, where $A, B$ run over $0,1,2,3$ and 5 ).

The energy-momentum tensor of the matter so induced is of a general nature but has well-defined properties (see Refs. 6 for discussion) consistent with those of

${ }^{a}$ This equation should be identical with Eq. (8) of Ref. 5. The latter however contains two typesetting mistakes, a missing $(\dot{a} / a)^{2}$-term and a missing overdot on one of the $\phi$-terms. Ref. 5 is also missing an equal sign between the first and second lines of Eq. (3), and the RHS of Eq. (17) in that paper should read $2 \ddot{a} / \dot{a}$ rather than $2 \ddot{a} / a$. 
the energy-momentum tensor $T_{i j}^{\phi}$ in Eq. (3). In this sense one can interpret both $4 \mathrm{D}$ matter and the scalar field in modified GST theory as manifestations of 5D geometry. More generally, one can consider homogeneous and isotropic extensions of the Robertson-Walker metric to a 5D manifold, $d^{(5)} s^{2}=d^{(4)} s^{2}+e^{\mu}\left(d x^{5}\right)^{2}$, where $d^{(4)} s^{2}$ is the 4D RW lipe element and $\mu$ is taken as a function of both $x^{0}=c t$ and the fifth coordinate $x^{5}$. IAs was first shown in Ref. 11 (see also Ref. 5), substitution of this metric into the $5 \mathrm{D}$ vacuum field equations leads to

$$
\begin{gathered}
\left(\frac{\dot{a}}{a}\right)^{2}+\frac{k c^{2}}{a^{2}}+\frac{1}{2} \frac{\dot{a}}{a} \dot{\mu}=0, \\
2 \frac{\ddot{a}}{a}+\left(\frac{\dot{a}}{a}\right)^{2}+\frac{k c^{2}}{a^{2}}+\frac{\ddot{\mu}}{2}+\frac{\dot{\mu}^{2}}{4}+\frac{\dot{a}}{a} \dot{\mu}=0, \\
\frac{\ddot{a}}{a}+\left(\frac{\dot{a}}{a}\right)^{2}+\frac{k c^{2}}{a^{2}}=0 .
\end{gathered}
$$

These results have the same form as the GST equations (5), (6) and (9) respectively; and in fact all six equations form a self-consistent set if the metric coefficient of the fifth coordinate satisfies $\dot{\mu}=2 \ddot{a} / \dot{a}$ (a result which played an important role in Ref. 5 but will not be needed here). The salient point here is that Eq. (12) from 5D STM theory gives the same result as the separable differential equation (9) in our modified GST theory, if the separability parameter $K$ in the latter is set to zero.

The solution of Eq. (12) is 11

$$
a=\sqrt{-k c^{2} t^{2}+\alpha t+\beta},
$$

where $\alpha$ and $\beta$ are constants. Differentiating twice, we find that the scale factor, Hubble parameter $H \equiv \dot{a} / a$ and deceleration parameter $q \equiv-a \ddot{a} / \dot{a}^{2}$ of the theory may be expressed in dimensionless form as follows

$$
\tilde{a}(\tilde{t})=\sqrt{-k \tilde{t}^{2}+\tilde{\alpha} \tilde{t}+\tilde{\beta}}, \quad \tilde{H}(\tilde{t})=\frac{\tilde{\alpha}-2 k \tilde{t}}{2 \tilde{a}^{2}}, \quad q(\tilde{t})=\frac{\tilde{\alpha}^{2} / 4+k \tilde{\beta}}{\tilde{H}^{2} \tilde{a}^{4}},
$$

where tildes denote dimensionless quantities and

$$
\tilde{t} \equiv H_{0} t, \quad \tilde{a} \equiv \frac{H_{0} a}{c}, \quad \tilde{H} \equiv \frac{H}{H_{0}}, \quad \tilde{\alpha} \equiv \frac{H_{0} \alpha}{c^{2}}, \quad \tilde{\beta} \equiv \frac{H_{0}^{2} \beta}{c^{2}} .
$$

We require that $\tilde{a}\left(\tilde{t}_{0}\right)=\tilde{a}_{0}, \tilde{H}\left(\tilde{t}_{0}\right)=1$, and $q\left(\tilde{t}_{0}\right)=q_{0}$ at the present time $\tilde{t}_{0}$, and also that the present phase of expansion begin in either a big bang $[\tilde{a}(0)=0]$ or a "big bounce" $[\tilde{H}(0)=0]$ at time zero. The theory then admits four classes of solutions (Table 1). Models I, II and III are all characterized by a big bang, as usual. For Model IV, one also finds that the size of the scale factor at the moment

\footnotetext{
${ }^{b}$ This new coordinate $x^{5}$ need not necessarily be lengthlike, as in traditional Kaluza-Klein theory, but could for instance be related to particle rest mass via $x^{5}=G m / c^{2}$ (Ref. 10) or other quantities (see Refs. 5, 6 for discussion).
} 
Table 1. Cosmological Model Parameters.

\begin{tabular}{ccccccc}
\hline Model & $k$ & $q_{0}$ & $\tilde{a}_{0}$ & $\tilde{\alpha}$ & $\tilde{\beta}$ & $\tilde{t}_{0}$ \\
\hline I & 0 & 1 & free & $2 \tilde{a}_{0}^{2}$ & 0 & $1 / 2$ \\
II & +1 & $q_{0}>1$ & $1 /\left(\sqrt{q_{0}-1}\right)$ & $2 \sqrt{q_{0}} \tilde{a}_{0}^{2}$ & 0 & $1 /\left(1+\sqrt{q_{0}}\right)$ \\
III & -1 & $0<q_{0}<1$ & $1 /\left(\sqrt{1-q_{0}}\right)$ & $2 \sqrt{q_{0}} \tilde{a}_{0}^{2}$ & 0 & $1 /\left(1+\sqrt{q_{0}}\right)$ \\
IV & -1 & $q_{0}<0$ & $1 /\left(\sqrt{1-q_{0}}\right)$ & 0 & $-q_{0} /\left(1-q_{0}\right)^{2}$ & $1 /\left(1-q_{0}\right)$ \\
\hline
\end{tabular}

of the big bounce is given by $\tilde{a}(0)=\tilde{\beta}^{1 / 2}=\sqrt{-q_{0}} \tilde{a}_{0}^{2}$. This tends to zero as $q_{0} \rightarrow 0$, so that one also recovers a big bang in this limit.

Current data on Hubble's constant and the age of the Universe imply that $H_{0} \geq 65 \mathrm{~km} \mathrm{~s}^{-1} \mathrm{Mpc}^{-1}$ and $t_{0} \geq 11 \mathrm{Gyr}, 12$ so that we may take $\tilde{t}_{0}>0.7$. This would rule out models of types I and II, which have $\tilde{t}_{0} \leq 0.5$. The evolution of the scale factor in all four models is plotted in Fig. 1, where we have adopted the values $q_{o}=2$ for Model II, $\tilde{t}_{0}=0.8$ for Model III, and $q_{0}=-0.5$ for Model IV.

The physical meaning of the deceleration parameter $q$ in scalar-tensor gravity differs somewhat from that in standard general relativity, and needs to be clarified before numerical boundary conditions can be applied. From the matter conservation law $T^{k} i ; k=0$ one obtains the usual expression for the energy density of matter,

$$
\epsilon=\epsilon_{\gamma} a^{-3(1+\gamma)},
$$

where $\gamma \equiv p / \epsilon$ and $\epsilon_{\gamma}=$ const. The energy density and pressure of the scalar field can be obtained from Eq. (3) and read

$$
\epsilon_{\phi}=\frac{\phi c^{2}}{8 \pi}\left(-3 \frac{\dot{a}}{a} \frac{\dot{\phi}}{\phi}+\frac{f_{\Lambda}}{\phi}\right), \quad p_{\phi}=\frac{\phi c^{2}}{8 \pi}\left(\frac{\ddot{\phi}}{\phi}+2 \frac{\dot{a}}{a} \frac{\dot{\phi}}{\phi}-\frac{f_{\Lambda}}{\phi}\right) .
$$

Eliminating $\phi$ from these two expressions, we find

$$
\epsilon_{\phi}-3 p_{\phi}=(3 \gamma-1) \epsilon_{\gamma} a^{-3(\gamma+1)} .
$$

Following the approach adopted in other quintessence-type theories, 1 one can define a ratio of $p_{\phi}$ to $\epsilon_{\phi}$ for the scalar field (analogous to that for matter) via

$$
p_{\phi} \equiv \gamma_{\phi} \epsilon_{\phi}
$$

where $\gamma_{\phi}$ is in general a function of $t$. More sophisticated equations of state are possible too. With Eq. (19), one finds for the deceleration parameter,

$$
q=\frac{(1+3 \gamma)}{2} \Omega_{m}+\frac{\left(1+3 \gamma_{\phi}\right)}{2} \Omega_{\phi},
$$

where the energy density parameters of matter and the scalar field are respectively

$$
\Omega_{m} \equiv \frac{8 \pi \epsilon}{3 H^{2} \phi c^{2}}, \quad \Omega_{\phi} \equiv \frac{8 \pi \epsilon_{\phi}}{3 H^{2} \phi c^{2}}=-\frac{1}{H} \frac{\dot{\phi}}{\phi}+\frac{f_{\Lambda}}{3 H^{2} \phi} .
$$


In the case $\phi=$ const., [or equivalently $\Lambda=$ const.] where the energy-momentum tensor of the scalar field reduces to that of a vacuum $\left(\gamma_{\phi}=\gamma_{\text {vac }}=-1\right)$ and we recover the standard expression for the deceleration parameter,

$$
q=\frac{(1+3 \gamma)}{2} \Omega_{m}-\Omega_{\mathrm{vac}} .
$$

Observations of galaxy clusters indicate that $\Omega_{m, 0}<0.5$, while analysis of fluctuations in the cosmic microwave background (CMB) gives $\Omega_{m, 0}+\Omega_{\Lambda, 0} \approx 1.1 \pm 0.1$ (see Ref. 12 for a recent review of the observational data). Both numbers together imply that $\Omega_{\Lambda, 0} \geq 0.5$, a result which agrees with data on distant supernovae. For dustlike $(\gamma=0)$ models with $\phi=$ const., we would then conclude from Eq. (22) that $q_{0}<-0.2$, which would restrict us to Model IV in our theory. For more general situations, we can say nothing about the value of $q_{0}$ at this stage, and either of Models III and IV are viable in principle.

\section{Evolution of the Scalar Field}

Integrating Eq. (8) with $\omega=\omega(\phi)$, we find with the help of Eq. (12) that

$$
\Lambda c^{2}=\frac{\omega}{2}\left(\frac{\dot{\phi}}{\phi}\right)^{2}+\frac{f_{\Lambda}}{\phi},
$$

where $f_{\Lambda}=$ const. Eq. (23) shows that in an RW universe with $\phi=$ const., $\Lambda$ is constant too. Alternatively, in Brans-Dicke (BD) theory with $\phi$ a power-law function of time and $\omega=$ const., the first term on the right-hand side of Eq. (23) goes as $t^{-2}$ (this behavior is common to other modified GST theoriest).

The scalar field $\phi(c t)$ is then obtained from either of Eqs. (5) or (6), with the help of Eq. (8) for $\partial \Lambda c^{2} / \partial \dot{\phi}^{2}$, Eq. (13) for $a$, Eq. (16) for $\epsilon$ (or $p$ ), and Eq. (23) for $\Lambda c^{2}$. The result is

$$
\dot{\phi}+\frac{\alpha^{2}+4 k \beta c^{2}}{2\left(-2 k c^{2} t+\alpha\right) a^{2}} \phi=\frac{2 a^{2}}{-2 k c^{2} t+\alpha}\left[\frac{8 \pi \epsilon_{\gamma}}{3 c^{2}} a^{-3(1+\gamma)}+\frac{f_{\Lambda}}{3}\right] .
$$

Eq. (24) is a linear differential equation of first order, and may be solved analytically for $\phi(t)$ in the cases $k=0, \pm 1$ and $\gamma=-1,1,1 / 3,0$. For $k=0$ we obtain

$$
\phi= \begin{cases}\frac{32 \pi \epsilon_{-1}}{15 \alpha^{2} c^{2}} a^{4}+\frac{4 f_{\Lambda}}{15 \alpha^{2}} a^{4}+\frac{\phi_{k}}{a} & \text { if } \gamma=-1 \\ -\frac{32 \pi \epsilon_{1}}{3 \alpha^{2} c^{2} a^{2}}+\frac{4 f_{\Lambda}}{15 \alpha^{2}} a^{4}+\frac{\phi_{k}}{a} & \text { if } \gamma=1 \\ \frac{32 \pi \epsilon_{1 / 3}}{3 \alpha^{2} c^{2}}+\frac{4 f_{\Lambda}}{15 \alpha^{2}} a^{4}+\frac{\phi_{k}}{a} & \text { if } \gamma=\frac{1}{3} \\ \frac{16 \pi \epsilon_{0} t}{3 \alpha c^{2} a}+\frac{4 f_{\Lambda}}{15 \alpha^{2}} a^{4}+\frac{\phi_{k}}{a} & \text { if } \gamma=0 .\end{cases}
$$


We solve Eq. (24) for $k= \pm 1$ as follows,

$$
\phi= \begin{cases}\frac{\mp 2 c^{2} t+\alpha}{a}\left(\frac{16 \pi \epsilon_{-1}}{3 c^{2}} \Phi+\frac{2 f_{\Lambda}}{3} \Phi+\phi_{k}\right) \quad \text { if } \gamma=-1 & \\ \frac{32 \pi \epsilon_{1}\left(-8 c^{4} t^{2} \pm 8 \alpha c^{2} t-\alpha^{2} \pm 4 \beta c^{2}\right)}{3 c^{2}\left(\alpha^{2} \pm 4 \beta c^{2}\right)^{2} a^{2}}+\frac{\mp 2 c^{2} t+\alpha}{a}\left(\frac{2 f_{\Lambda}}{3} \Phi+\phi_{k}\right) & \text { if } \gamma=1 \\ \frac{32 \pi \epsilon_{1 / 3}}{3 c^{2}\left(\alpha^{2} \pm 4 \beta c^{2}\right)}+\frac{\mp 2 c^{2} t+\alpha}{a}\left(\frac{2 f_{\Lambda}}{3} \Phi+\phi_{k}\right) \quad \text { if } \gamma=\frac{1}{3} & \\ \pm \frac{8 \pi \epsilon_{0}}{3 c^{4} a}+\frac{\mp 2 c^{2} t+\alpha}{a}\left(\frac{2 f_{\Lambda}}{3} \Phi+\phi_{k}\right) & \text { if } \gamma=0,\end{cases}
$$

where

$$
\Phi \equiv \frac{a^{3}}{-4 c^{4} t \pm 2 \alpha c^{2}}+\frac{3}{16 c^{4}}\left[\left(\mp 2 c^{2} t+\alpha\right) a-\frac{\alpha^{2} \pm 4 \beta c^{2}}{2} I_{F}\right]
$$

and

$$
I_{F} \equiv \begin{cases}-\frac{1}{c} \arcsin \frac{-2 c^{2} t+\alpha}{\sqrt{\alpha^{2}+4 \beta c^{2}}} & \text { if } k=+1 \\ \frac{1}{2 c} \ln \left|\frac{2 c^{2} t+\alpha+2 c a}{2 c^{2} t+\alpha-2 c a}\right| & \text { if } k=-1 .\end{cases}
$$

To obtain a quantitative idea of the evolution of the scalar field with time, we need the parameter $\epsilon_{\gamma}$ in Eqs. (25) and (26). Application of Eq. (16) at the present time with the boundary condition $\epsilon\left(t_{0}\right)=c^{2} \rho_{0}=c^{2} \rho_{\text {crit }} \Omega_{m, 0}$ leads to

$$
\begin{aligned}
& \epsilon_{-1}=\left(\frac{3 H_{0}^{2} c^{2}}{8 \pi G_{0}}\right) \Omega_{m, 0}, \epsilon_{1}=\left(\frac{3 c^{8}}{8 \pi G_{0} H_{0}^{4}}\right) \Omega_{m, 0} \tilde{a}_{0}^{6}, \\
& \epsilon_{1 / 3}=\left(\frac{3 c^{6}}{8 \pi G_{0} H_{0}^{2}}\right) \Omega_{m, 0} \tilde{a}_{0}^{4}, \quad \epsilon_{0}=\left(\frac{3 c^{5}}{8 \pi G_{0} H_{0}}\right) \Omega_{m, 0} \tilde{a}_{0}^{3},
\end{aligned}
$$

where the present critical density $\rho_{\text {crit }} \equiv 3 H_{0}^{2} / 8 \pi G_{0}$ and $G_{0}$ is the present value of Newton's gravitational constant (which varies roughly as $1 / \phi$ in scalar-tensor theories). Of these parameters, only the last is relevant to the present (dustlike) universe. In a more sophisticated model one would allow for different epochs (characterized by different values of $\gamma$ ) and evaluate Eq. (16) across the phase transitions between them, enforcing continuity to obtain realistic values of $\epsilon_{\gamma}$.

We proceed to define a dimensionless scalar field via $\tilde{\phi} \equiv\left(G_{0} / \Omega_{m, 0}\right) \phi$. With $\epsilon_{\gamma}$ as given in Eqs. (29), this is found to consist of three components,

$$
\tilde{\phi}=\tilde{\phi}_{1}+\tilde{\phi}_{2}+\tilde{\phi}_{3}
$$

where $\tilde{\phi}_{1}, \tilde{\phi}_{2}$ and $\tilde{\phi}_{3}$ take simple analytic forms, depending on the model parameters (see Appendix). Figs. 2(a)-(d) show the evolution of the scalar field $\tilde{\phi}$ in Models I-IV respectively, using the same values for $q_{0}$ as those in Fig. 1, and values of $\tilde{f}_{\Lambda}$ and $\tilde{\phi}_{k}$ as marked beside the curves. 


\section{Evolution of the Cosmological Term}

The cosmological term is found from Eq. (23) and may be expressed in the usual form of a dimensionless vacuum energy density parameter

$$
\Omega_{\Lambda} \equiv \frac{\Lambda c^{2}}{3 H_{0}^{2}}=\frac{\omega}{6}\left(\frac{1}{H_{0}} \frac{\dot{\tilde{\phi}}}{\tilde{\phi}}\right)^{2}+\frac{5 \tilde{f}_{\Lambda}}{\tilde{\phi}} .
$$

(Note that this is not the same as $\Omega_{\phi}$.) At the present time, $\Omega_{\Lambda}=\Omega_{\Lambda, 0}$ is the quantity measured by observers using, e.g., the magnitude-redshift relation for distant supernovae. Eq. (31) cannot be evaluated without a specific functional form for $\omega(\phi)$. Let us consider to begin with the case in which $\omega=\omega_{0}=$ const., and the simplest models in which $\tilde{f}_{\Lambda}=\tilde{\phi}_{k}=0$. Taking $\gamma=0$ (dust) and $k=0$ (Model I), we find from Eq. (31) that

$$
\Omega_{\Lambda, 0}=\frac{\omega_{0}}{6}\left(\frac{1-\tilde{t}_{0}}{\tilde{t}_{0}}\right)^{2}=\frac{\omega_{0}}{6}
$$

since $\tilde{t}_{0}=1 / 2$ for Model I. Using the observational upper limit $\Omega_{\Lambda, 0} \leq 1.12$ we find that $\omega_{0}<7$ which violates the current experimental lower bound $\left(\omega_{0} \geq 600\right)$ on this quantity. 13 This means that, at least in the case $k=0$ with $\omega=$ const., we cannot have both $\tilde{f}_{\Lambda}$ and $\tilde{\phi}_{k}$ equal to zero.

Alternatively, we can use Eq. (31) to constrain the values of $\tilde{f}_{\Lambda}$ and $\tilde{\phi}_{k}$ if we impose the boundary conditions $\Omega_{\Lambda}=\Omega_{\Lambda, 0}$ and $\omega=\omega_{0}=600$ (say). For Model I $(k=0)$ as the simplest case, we find on differentiating Eq. (30) that this procedure gives a quadratic for $\tilde{f}_{\Lambda}$ (if $\tilde{\phi}_{k}$ is held constant) or $\tilde{\phi}_{k}$ (if $\tilde{f}_{\Lambda}$ is held constant). In particular, if $\tilde{f}_{\Lambda}=0$, then $\tilde{\phi}_{k}=0.418$ and 0.598 both give $\Omega_{\Lambda, 0}=0.8$ at the present time (with minima in the past and future respectively). Similarly, if $\tilde{\phi}_{k}=0$, then the same thing occurs for $\tilde{f}_{\Lambda}=-0.140$ and -0.110 . This second possibility however involves a negative cosmological term in the near past (or future), which is strongly disfavored by observation as it would shorten the age of the Universe. The implication is that $\tilde{\phi}_{k}$, which arises as a constant of integration in Eqs. (25) and (26), is probably nonzero in models of this kind if they are to be realistic. This scalar-field theory, in other words, is of the "chaptic" kind, in which the field begins to roll from an (arbitrary) nonzero initial value 14

We show four representative solutions of Eq. (31) in Fig. 3, which assumes Model I $(k=0)$ and $\omega_{0}=600$. These have been fit to $\Omega_{\Lambda, 0}=0.8$ at the present time, as suggested by current experimental data.12 Simplest is the $\tilde{f}_{\Lambda}=0$ solution, but all are natural in the sense of having dimensionless parameters (other than $\omega_{0}$ ) of order unity. All models, moreover, show a steep increase in $\Omega_{\Lambda}$ in the past direction. This behavior is generic to constant- $\omega$ solutions of Model II, III and IV as well as those of Model I. The modified GST theory, therefore, provides one possible mechanism for addressing the problem of the cosmological "constant" 3 Any such increase during the nucleosynthesis and decoupling eras, however, must satisfy 
constraints based on light element synthesist 15 and the observed spectrum of CMB anisotropies 16 In a realistic GST, the behavior of $\Omega_{\Lambda}$ is probably more complicated than that shown in Fig. 3, and $\omega(\phi)$ will almost certainly not be constant.

Let us accordingly consider some aspects of models in which $\omega(\phi) \neq$ const. We focus on the matter and radiation eras, since these may be constrained by observational cosmology and again take the simplest case with $k=0$ and $\beta=0$. During the matter era $(\gamma=0)$, the cosmological term is given by Eq. (23) as

$$
\Lambda_{m} c^{2}=\frac{1}{8 t_{m}^{2}}\left[\omega\left(\phi_{m}\right)\left(\frac{1+4 E_{1} t_{m}^{3 / 2}-E_{2} / t_{m}}{1+E t_{m}^{3 / 2}+E_{2} / t_{m}}\right)^{2}+\frac{30 E_{1} t_{m}^{3 / 2}}{1+E_{1} t_{m}^{3 / 2}+E_{2} / t_{m}}\right],
$$

where

$$
E_{1} \equiv \frac{f_{\Lambda} \alpha^{3 / 2} c^{2}}{20 \pi \epsilon_{0}}, \quad E_{2} \equiv \frac{3 \phi_{k} \alpha c^{2}}{16 \pi \epsilon_{0}}
$$

Here $\epsilon_{0}$ is the value of $\epsilon_{\gamma}$ at $\gamma=0$ in Eq. (16) [see Eq. (29)]. The energy density parameter of matter may be obtained from Eq. (21)

$$
\Omega_{m}=\frac{2}{1+E_{1} t_{m}^{3 / 2}+E_{2} / t_{m}}
$$

Its present value, $\Omega_{m, 0}=2 /\left(1+E_{1} t_{0}^{3 / 2}+E_{2} / t_{0}\right)$, is constrained experimentally to the range $0.01 \leq \Omega_{m, 0} \leq 0.5 .12$

During the radiation era $(\gamma=1 / 3)$, on the other hand,

$$
\Lambda_{r} c^{2}=\frac{1}{4 t_{r}^{2}}\left[8 \omega\left(\phi_{r}\right)\left(\frac{B_{1} t_{r}^{2}-B_{2} / 4 t_{r}^{1 / 2}}{1+B_{1} t_{r}^{2}+B_{2} / t_{r}^{1 / 2}}\right)^{2}+\frac{15 B_{1} t_{r}^{2}}{1+B_{1} t_{r}^{2}+B_{2} / t_{r}^{1 / 2}}\right],
$$

where

$$
B_{1} t_{r}^{2} \equiv \frac{f_{\Lambda} \alpha^{2} c^{2}}{40 \pi \epsilon_{1 / 3}} t_{r}^{2}=\frac{\epsilon_{m}}{2 \epsilon_{r}} E_{1} t_{m}^{3 / 2}, \quad \frac{B_{2}}{t_{r}^{1 / 2}} \equiv \frac{3 \phi_{k} \alpha^{3 / 2} c^{2}}{32 \pi \epsilon_{1 / 3} t_{r}^{1 / 2}}=\frac{\epsilon_{m}}{2 \epsilon_{r}}\left(\frac{t_{m}}{t_{r}}\right)^{5 / 2} \frac{E_{2}}{t_{m}}
$$

If we take $f_{\Lambda}=0$ as above (i.e., $E_{1}=0$ and $B_{1}=0$ ), then the cosmological term at nucleosynthesis time $t=t_{n}$ is given by Eq. (36) as

$$
\Lambda_{n} c^{2}=\frac{\omega\left(\phi_{n}\right)}{8 t_{n}^{2}}\left(\frac{B_{2} / t_{n}^{1 / 2}}{1+B_{2} / t_{n}^{1 / 2}}\right)^{2} \sim \frac{\omega\left(\phi_{n}\right)}{8 t_{n}^{2}},
$$

where $B_{2} / t_{n}^{1 / 2} \gg 1$ from Eqs. (34) and (37), since $\epsilon_{n} \sim 10^{30} \epsilon_{0}, t_{0} \sim 10^{15} t_{n}$ and $E_{2} / t_{0} \sim 4$. At decoupling time $t=t_{d}$, finally, Eq. (33) gives

$$
\Lambda_{d} c^{2}=\frac{\omega\left(\phi_{d}\right)}{8 t_{d}^{2}}\left(\frac{1-E_{2} / t_{d}}{1+E_{2} / t_{d}}\right)^{2} \sim \frac{\omega\left(\phi_{d}\right)}{8 t_{d}^{2}}
$$


where $E_{2} / t_{d}=\left(2 / \Omega_{m, 0}-1\right)\left(t_{0} / t_{d}\right) \gg 1$ from Eq. (34), since $t_{0} \sim 10^{5} t_{d}$. These expressions can in principle be used to constrain the behavior of $\omega(\phi)$ during the radiation era via nucleosynthesis considerations 15 and observations of the power spectrum of CMB fluctuations.16

\section{Discussion}

We have modified the generalized scalar-tensor theory by taking the cosmological term as a function not only of the scalar field but of its gradient as well. This means that we effectively interpret both the second and third terms in Wagoner's Lagrangian density [Eq. (2) in Ref. 1] as a single variable cosmological term. In the context of higher-dimensional theories this may be justified by considering the origin of the scalar field, which is infuced in $4 \mathrm{D}$ spacetime by the geometry of the empty higher-dimensional universe 17 The scalar field and cosmological term can in this sense be recognized together as a single geometrical entity. We in the $4 \mathrm{D}$ universe then study this as constant for de Sitter models, or as temperature-dependent in spontaneous symmetry-breaking models 18 or as kinetic energy-like in connection with the age 19 or dark matter problems. 20 A recent comprehensive discussion of the cosmological term has been given by Sahni and Starobinsky.21

We have previously explored the relationship between the modified GST theory (11) and 5D STM theory in Ref. 5. There, an identification of the extra part of the higher-dimensional metric tensor in terms of the scalar field $\phi$ enabled us to obtain solutions for $\Lambda$ relevant to vacuum-dominated or stiff matter-like phases in the history of the Universe. In the present paper, a different ansatz (8) has led to a wider class of solutions which can be used to describe the radiation and matter-dominated eras. As seen from Eq. (23) and the solutions (25) and (26), the cosmological term evolves differently in each era because $\phi$ depends explicitly on $\gamma$. This presents a mechanism for generating new cosmological terms during successive cosmological phase transitions.

This work has made use of STM theory, because it is one of our motivations to explore the relevance of higher-dimensional physics to 4D cosmology. But Eq. (9) admits a second class of solutions for the evolution of the scale factor when we do not make use of Eq. (12) from STM theory to set Eq. (9) to zero. Fukui et al. 6 have used these to model inflation in the early universe - something for which the present set of solutions is not suited, as seen from the form of Eq. (13). Taken together, both classes of solutions can allow us to follow the evolution of the cosmological term throughout the history of the Universe. These and other aspects of modified GST theory will be pursued further elsewhere.

\section{Acknowledgements}

T. F. is grateful for the hospitality of the Department of Physics, University of Waterloo, while staying on the research program of Dokkyo University. J. M. O. is supported by a Postdoctoral Fellowship from the Japan Society for the Promotion 
of Science (JSPS).

\section{References}

[1] P. G. Bergmann, Int. J. Theor. Phys. 1, 25 (1968);

R. V. Wagoner, Phys. Rev. D1, 3209 (1970).

[2] B. Ratra and P. J. E. Peebles, Phys. Rev. D37, 3407 (1988);

J. D. Barrow and K. Maeda, Nucl. Phys. B341, 294 (1990);

J. A. Frieman, C. T. Hill, A. Stebbins and I. Waga, Phys. Rev. Lett. 75, 2077 (1995), astro-ph/9505060.

[3] S. Weinberg, Rev. Mod. Phys. 61, 1 (1989);

Y. Fujii and T. Nishioka, Phys. Rev. D42, 361 (1990);

J. M. Overduin and F. I. Cooperstock, Phys. Rev. D58, 043506 (1998), astro$\mathrm{ph} / 9805260$;

T. Fukui, Int. J. Mod. Phys. D10, 893 (2001).

[4] R. P. Caldwell, R. Dave and P. J. Steinhardt, Phys. Rev. Lett. 80, 1582 (1998), astro$\mathrm{ph} / 9708069$;

L. Wang, R. R. Caldwell, J. P. Ostriker and P. J. Steinhardt, Astrophys. J. 530, 17 (2000), astro-ph/9901388.

[5] T. Fukui and J. M. Overduin, Gen. Rel. Grav. 31, 1159 (1999).

[6] J. M. Overduin and P. S. Wessson, Phys. Rep. 283, 283 (1997), gr-qc/9805018;

P. S. Wesson, Space-Time-Matter (World Scientific, Singapore, 1999).

[7] M. Endō and T. Fukui, Gen. Rel. Grav. 8, 833 (1977);

K. Arai, M. Hashimoto and T. Fukui, Astron. Astrophys. 179, 17 (1987);

T. Etoh, M. Hashimoto, K. Arai and S. Fujimoto, Astron. Astrophys. 325, 893 (1997).

[8] T. Fukui, K. Arai and M. Hashimoto, Class. Quant. Grav. 18, 2087 (2001).

[9] S. Weinberg, Gravitation and Cosmology (Wiley, New York, 1972), p. 159.

[10] P. S. Wesson, Astron. Astrophys. 119, 145 (1983).

[11] A. Banerjee, B. K. Bhui and S. Chatterjee, Astron. Astrophys. 232, 305 (1990).

[12] J. Overduin and W. Priester, Naturwissenschaften 88, 229 (2001), astro-ph/0101484.

[13] C. M. Will, Theory and Experiment in Gravitational Physics (CUP, Cambridge, 1993), p. 335 .

[14] A. D. Linde, Phys. Lett. 129B, 177 (1983).

[15] M. Birkel and S. Sarkar, Astropart. Phys. 6, 197 (1997), astro-ph/9605055;

T. Damour and B. Pichon, Phys. Rev. D59, 123502 (1999), astro-ph/9807176.

[16] V. Silveira and I. Waga, Phys. Rev. D50, 4890 (1994);

V. Silveira and I. Waga, Phys. Rev. D56, 4625 (1997);

P. Brax, J. Martin and A. Riazuelo, Phys. Rev. D62, 103505 (2000), astro-ph/0005428.

[17] P. Jordan, in Cosmological Models, Proc. International Summer Institute, Lisbon, September 1963 (Centro de Cálculo Cientifico Instituto Gulbenkian de Ciência, Lisboa, 1964), p. 117.

[18] A. D. Linde, JETP Lett. 19, 183 (1974).

[19] W. Moffat, Phys. Lett. B357, 526 (1995), gr-qc/9412010.

[20] C. Armendáriz-Picón, T. Damour and V. Mukhanov, Phys. Lett. B458, 209 (1999), hep-th/9904075.

[21] V. Sahni and A. Starobinsky, Int. J. Mod. Phys. D9, 373 (2000), astro-ph/9904398. 


\section{Appendix A}

In this Appendix we give expressions for the components $\tilde{\phi}_{1}, \tilde{\phi}_{2}$ and $\tilde{\phi}_{3}$ of the dimensionless scalar field, Eq. (30), as obtained from Eqs. (25) and (26) and plotted in Figs. 2 of the main text. For Model I:

$$
\begin{aligned}
& \tilde{\phi}_{1}=\left\{\begin{array}{l}
H_{0} t\left(\frac{a}{a_{0}}\right)^{-1} \quad \text { if } \gamma=0 \\
1 \quad \text { if } \gamma=1 / 3 \\
-\left(\frac{a}{a_{0}}\right)^{-2} \quad \text { if } \gamma=1 \\
\frac{1}{5}\left(\frac{a}{a_{0}}\right)^{4} \quad \text { if } \gamma=-1 ;
\end{array}\right. \\
& \tilde{\phi}_{2}=5 \tilde{f}_{\Lambda} \tilde{\phi}_{1} \quad\left(\text { with } \tilde{\phi}_{1} \text { as for } \gamma=-1 \text { case) } ;\right. \\
& \tilde{\phi}_{3}=\tilde{\phi}_{k}\left(\frac{a}{a_{0}}\right)^{-1} .
\end{aligned}
$$

For Model II:

$$
\begin{aligned}
& \tilde{\phi}_{1}=\left\{\begin{array}{l}
\frac{1}{q_{0}-1}\left(\frac{a}{a_{0}}\right)^{-1} \quad \text { if } \gamma=0 \\
\frac{1}{q_{0}} \quad \text { if } \gamma=1 / 3 \\
\frac{1}{q_{0}}\left(\frac{a}{a_{0}}\right)^{-2}\left(1-2 \xi^{2}\right) \quad \text { if } \gamma=1 \\
\frac{1}{q_{0}-1}\left(\frac{a}{a_{0}}\right)^{2}\left(1+\frac{3}{2} \zeta^{2}+\frac{3 \zeta^{3}}{2 \xi^{2}} \arcsin \xi\right) \quad \text { if } \gamma=-1 ;
\end{array}\right. \\
& \tilde{\phi}_{2}=5 \tilde{f}_{\Lambda} \tilde{\phi}_{1} \quad\left(\text { with } \tilde{\phi}_{1} \text { as for } \gamma=-1 \text { case }\right) ; \\
& \tilde{\phi}_{3}=2 \tilde{\phi}_{k} \zeta
\end{aligned}
$$


For Model III:

$$
\begin{aligned}
& \tilde{\phi}_{1}=\left\{\begin{array}{l}
\frac{1}{q_{0}-1}\left(\frac{a}{a_{0}}\right)^{-1} \quad \text { if } \gamma=0 \\
\frac{1}{q_{0}} \quad \text { if } \gamma=1 / 3 \\
\frac{1}{q_{0}}\left(\frac{a}{a_{0}}\right)^{-2}\left(1-2 \xi^{2}\right) \quad \text { if } \gamma=1 \\
\frac{1}{q_{0}-1}\left(\frac{a}{a_{0}}\right)^{2}\left(1-\frac{3}{2} \zeta^{2}+\frac{3 \zeta^{3}}{4 \xi^{2}} \ln \left|\frac{\zeta+1}{\zeta-1}\right|\right) \quad \text { if } \gamma=-1 ;
\end{array}\right. \\
& \tilde{\phi}_{2}=5 \tilde{f}_{\Lambda} \tilde{\phi}_{1} \quad\left(\text { with } \tilde{\phi}_{1} \text { as for } \gamma=-1 \text { case }\right) ; \\
& \tilde{\phi}_{3}=2 \tilde{\phi}_{k} \zeta .
\end{aligned}
$$

For Model IV:

$$
\begin{aligned}
& \tilde{\phi}_{1}=\left\{\begin{array}{l}
\frac{1}{q_{0}-1}\left(\frac{a}{a_{0}}\right)^{-1} \quad \text { if } \gamma=0 \\
\frac{1}{q_{0}} \quad \text { if } \gamma=1 / 3 \\
\frac{1}{q_{0}}\left(\frac{a}{a_{0}}\right)^{-2}\left(1+2 \eta^{2}\right) \quad \text { if } \gamma=1 \\
\frac{1}{q_{0}-1}\left(\frac{a}{a_{0}}\right)^{2}\left(1-\frac{3}{2} \lambda^{2}-\frac{3 \lambda^{3}}{4 \eta^{2}} \ln \left|\frac{\lambda+1}{\lambda-1}\right|\right) \quad \text { if } \gamma=-1 ;
\end{array}\right. \\
& \tilde{\phi}_{2}=5 \tilde{f}_{\Lambda} \tilde{\phi}_{1} \quad\left(\text { with } \tilde{\phi}_{1} \text { as for } \gamma=-1 \text { case }\right) ; \\
& \tilde{\phi}_{3}=2 \tilde{\phi}_{k} \lambda .
\end{aligned}
$$

In these equations we have introduced four new parameters

$$
\begin{aligned}
& \xi \equiv 1-\left(1-\frac{1}{\sqrt{q_{0}}}\right) \frac{t}{t_{0}}, \quad \zeta \equiv \xi \sqrt{\frac{q_{0}}{\left|q_{0}-1\right|}}\left(\frac{a}{a_{0}}\right)^{-1}, \\
& \eta \equiv\left(\frac{1}{\sqrt{-q_{0}}}\right) \frac{t}{t_{0}}, \quad \lambda \equiv \frac{1}{\sqrt{1-q_{0}}}\left(\frac{a}{a_{0}}\right)^{-1} \frac{t}{t_{0}},
\end{aligned}
$$

and additionally defined two dimensionless free parameters $\tilde{f}_{\Lambda} \equiv\left(G_{0} / 15 H_{0}^{2} \Omega_{m, 0}\right) f_{\Lambda}$ and $\tilde{\phi}_{k} \equiv\left(G_{0} / a_{0} \Omega_{m, 0}\right) \phi_{k}$ (if $k=0$ ) or $\tilde{\phi}_{k} \equiv\left(c G_{0} / \Omega_{m, 0}\right) \phi_{k}$ (if $k= \pm 1$ ). In a natural theory one expects both of these parameters to take values of order unity (no finetuning). As discussed in Section 5 of the main text, this expectation appears to be borne out for the simplest models. 